\title{
The quest for companions to post-common envelope binaries
}

\section{A reexamination of $\mathrm{HW}$ Virginis}

\author{
K. Beuermann ${ }^{1}$, S. Dreizler ${ }^{1}$, F. V. Hessman ${ }^{1}$, and J. Deller ${ }^{1,2}$ \\ ${ }^{1}$ Institut für Astrophysik, Georg-August-Universität, Friedrich-Hund-Platz 1, 37077 Göttingen, Germany \\ e-mail: beuermann@astro.physik.uni-goettingen.de \\ 2 Centre for Astrophysics and Planetary Science, University of Kent, Canterbury, CT2 7NH, UK
}

Received 11 April 2012 / Accepted 11 June 2012

\begin{abstract}
We report new mid-eclipse times of the short-period sdB/dM binary HW Virginis, which differ substantially from the times predicted by a previous model. The proposed orbits of the two planets in that model are found to be unstable. We present a new secularly stable solution, which involves two companions orbiting HW Vir with periods of $12.7 \mathrm{yr}$ and $55 \pm 15 \mathrm{yr}$. For orbits coplanar with the binary, the inner companion is a giant planet with mass $M_{3} \sin i_{3} \simeq 14 M_{\text {Jup }}$ and the outer one a brown dwarf or low-mass star with a mass of $M_{4} \sin i_{4}=30-120 M_{\mathrm{Jup}}$. Using the mercury6 code, we find that such a system would be stable over more than $10^{7} \mathrm{yr}$, in spite of the sizeable interaction. Our model fits the observed eclipse-time variations by the light-travel time effect alone, without invoking any additional process, and provides support for the planetary hypothesis of the eclipse-time variations in close binaries. The signature of non-Keplerian orbits may be visible in the data.
\end{abstract}

Key words. planets and satellites: detection - binaries: close - binaries: eclipsing - subdwarfs - stars: individual: HW Virginis

\section{Introduction}

Periodic or quasiperiodic variations of the mid-eclipse times in close binaries have been observed for decades and variously ascribed to activity cycles of the secondary star (Applegate 1992), apsidal motion (Todoran 1972), or the response to a third body orbiting the binary (Nather \& Robinson 1974). More recently, Lee et al. (2009) assigned the complex O-C (observed minus calculated) eclipse-time variations in the detached $\mathrm{sdB} / \mathrm{dM}$ binary HW Vir with a 2.8-h orbital period to the light-travel time (LTT) effect caused by the orbital motion of two giant planets. Thereafter, an increasing number of eclipsing post-common envelope binaries (PCEB), both with sdB and white-dwarf primaries, were proposed as having eclipse-time variations possibly due to planetary or brown-dwarf companions. Systems with one proposed companion include HS0705+67, DPLeo, HS2231+24, and NSVS14256825 (Qian et al. 2009, 2010a,b; Beuermann et al. 2011, 2012). Further PCEB that may harbor more than one companion are NN Ser (Beuermann et al. 2010), UZ For (Potter et al. 2011), RR Cae and NY Vir (Qian et al. 2012a,b), QS Vir (Parsons et al. 2010), and HU Aqr (Qian et al. 2011; Hinse et al. 2012).

The question of secular stability does not arise for binaries with a single planet, but must be considered for the proposed systems of companions. Such analyses were not included in most original publications, with the exception of the two planets that orbit NN Ser, which represent a stable resonant pair (Beuermann et al. 2010). The system suggested by Qian et al. (2011) for HU Aqr, on the other hand, was shown to be secularly unstable (Horner et al. 2011; Wittenmyer et al. 2012), although a different stable solution may exist (Hinse et al. 2012). To be sure, the planetary hypothesis of the observed eclipse time variations has met with some scepticism (e.g. Wittenmyer et al. 2012), but a sufficiently well-defined alternative is not in sight.

A more general critique of the models of Lee et al. (2009) and Qian et al. (2011) relates to their combining the LTT effect with a long-term period decrease on a time scale of $\tau=$ $P / \dot{P} \simeq 10^{7}$ yr, supposedly produced by some other mechanism. Gravitational radiation leads to a period decrease, but is entirely negligible with $\tau \simeq 3 \times 10^{9} \mathrm{yr}$. Magnetic braking is a more adequate contender for a long-term period decrease, but cannot explain the recent period increase in HW Vir. Applegate's (1992) mechanism allows variations of both signs, but most authors agree that it is too feeble to produce the large observed eclipsetime variations (e.g. Brinkworth et al. 2006; Watson \& Marsh 2010). If, however, a so far unknown super-Applegate effect were operative in close binaries, it could conceivably account for the entire observed effect and render the arbitrary division into variations produced by different mechanisms meaningless. Indeed, it is the spectre of a super-Applegate mechanism that has historically prevented us from considering in close binary systems what, for unevolved single stars, has become the default situation: complex planetary systems (Lovis et al. 2011). The discovery by Kepler of several circumbinary planetary systems in non-evolved eclipsing binaries (Doyle et al. 2011; Welsh et al. 2012) shows us that it is not unreasonable to consider the same for evolved binaries, although their planetary systems possibly do not not survive the common-envelope phase unscathed. In this light, the proposed circumbinary planetary systems detected by the LTT method must be carefully re-scrutinized.

Here, we present new mid-eclipse times for HW Vir that deviate significantly from the Lee et al. (2009) prediction. We show that the planetary system proposed by Lee et al. is secularly unstable and, therefore, untenable. We find that a secularly stable 
Table 1. New primary mid-eclipse times $T_{\text {ecl }}$ of HW Vir and $\mathrm{O}-\mathrm{C}_{\text {lin, } 1}$ residuals for the MONET/N data relative to the ephemeris of Eq. (1).

\begin{tabular}{ccccc}
\hline \hline Cycle & $\begin{array}{c}\text { BJD(TT) } \\
2400000+\end{array}$ & $\begin{array}{c}\text { Error } \\
\text { (days) }\end{array}$ & $\begin{array}{c}\text { O-C } \mathrm{C}_{\text {lin, } 1} \\
\text { (days) }\end{array}$ & Origin \\
\hline 75123 & 54498.877674 & 0.000014 & N.A. & AAVSO \\
75890 & 54588.401364 & 0.000040 & N.A. & AAVSO \\
76004 & 54601.707367 & 0.000016 & N.A. & AAVSO \\
76063 & 54608.593786 & 0.000019 & N.A. & AAVSO \\
76089 & 54611.628553 & 0.000012 & N.A. & AAVSO \\
78062 & 54841.916149 & 0.000010 & N.A. & AAVSO \\
84077 & 55543.984048 & 0.000008 & -0.000007 & MONET/N \\
84120 & 55549.003005 & 0.000008 & 0.000009 & MONET/N \\
84180 & 55556.006176 & 0.000009 & 0.000006 & MONET/N \\
84411 & 55582.968393 & 0.000009 & 0.000006 & MONET/N \\
84428 & 55584.952622 & 0.000010 & 0.000003 & MONET/N \\
84488 & 55591.955807 & 0.000010 & 0.000014 & MONET/N \\
84497 & 55593.006274 & 0.000007 & 0.000005 & MONET/N \\
84600 & 55605.028372 & 0.000007 & -0.000010 & MONET/N \\
84608 & 55605.962117 & 0.000015 & -0.000022 & MONET/N \\
84693 & 55615.883298 & 0.000007 & -0.000004 & MONET/N \\
84863 & 55635.725619 & 0.000006 & -0.000007 & MONET/N \\
84967 & 55647.864460 & 0.000007 & 0.000001 & MONET/N \\
84976 & 55648.914932 & 0.000007 & -0.000004 & MONET/N \\
85026 & 55654.750921 & 0.000006 & 0.000007 & MONET/N \\
85249 & 55680.779371 & 0.000007 & -0.000003 & MONET/N \\
85266 & 55682.763597 & 0.000015 & -0.000009 & MONET/N \\
87093 & 55896.010239 & 0.000007 & 0.000005 & MONET/N \\
87589 & 55953.903110 & 0.000018 & -0.000023 & MONET/N \\
87624 & 55957.988315 & 0.000007 & -0.000003 & MONET/N \\
87787 & 55977.013609 & 0.000007 & 0.000004 & MONET/N \\
\hline & & & &
\end{tabular}

two-companion model can be devised, in which the observed eclipse-time variations are due to the LTT effect alone.

\section{The data base}

Starting in December 2010, we monitored the $V=10.9$ mag binary HW Vir with the MONET/North telescope at the University of Texas' McDonald Observatory via the MONET browserbased remote-observing interface. The photometric data were taken with an Apogee ALTA E47+ $1 \mathrm{k} \times 1 \mathrm{k}$ CCD camera mostly in the $I_{\mathrm{c}}$-band with exposure times of 10 or $20 \mathrm{~s}$. Since there is no suitable comparison star in our $5^{\prime} \times 5^{\prime}$ field, we included only observations obtained under photometric conditions. The light curves were analyzed using the heuristic mathematical model described in Paper II of this series (Beuermann et al. 2012). Table 1 lists the 20 new primary mid-eclipse times along with their formal $1-\sigma$ errors, which range from 0.5 to $1.6 \mathrm{~s}$. Fitting the MONET/N mid-eclipse times alone yields the linear ephemeris valid in $2010 / 2012$

$$
T_{\mathrm{ecl}}=\mathrm{BJD}(\mathrm{TT}) 2455543.984055(2)+0.116719555(2) E .
$$

The residuals from Eq. (1) are listed in Table 1 as $\mathrm{O}-\mathrm{C}_{\text {lin, }, 1}$. Their rms value of $0.8 \mathrm{~s}$ is consistent with the errors obtained from the formal fits to the light curves. Because of the effects of the additional bodies in the system, the period of Eq. (1) is not necessarily identical to the binary period.

A large body of primary and secondary mid-eclipse times of HW Vir is available in the literature. The SAAO group monitored HW Vir between 1984 and 2002 (Marang \& Kilkenny 1989) and Kilkenny et al. (1991, 1994, 2000, 2003), reporting a total of 111 primary mid-eclipse times with errors mostly as small as 2 s. The measurements of Lee et al. (2009) overlap with the SAAO data and extend the coverage to 2009, with

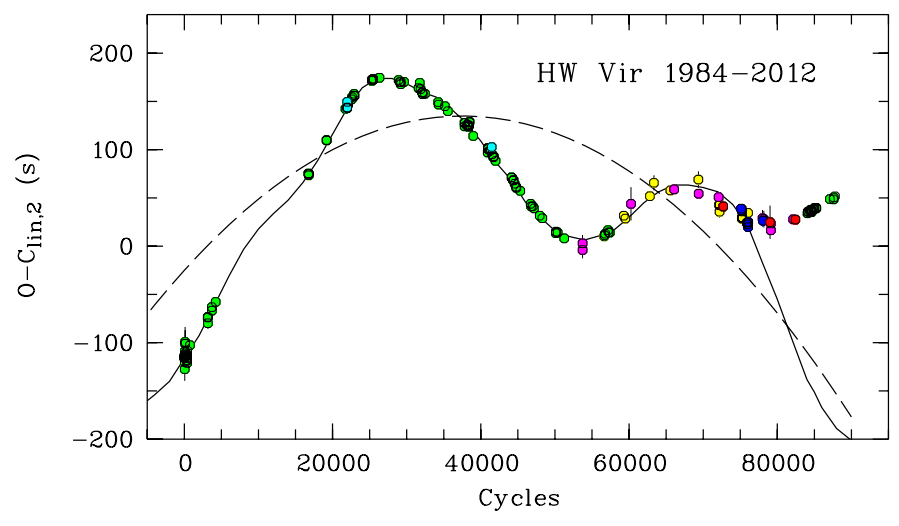

Fig. 1. $\mathrm{O}-\mathrm{C}_{\mathrm{lin}, 2}$ residuals of the mid-eclipse times from the linear ephemeris used by Lee et al. (2009) along with their model curves for the two-companion model (solid) and the underlying quadratic ephemeris (dashed). The data are from SAAO (green), Wood et al. (cyan blue), Lee et al. (yellow), BAV and VSNET (magenta), AAVSO (blue), BRNO (red), and MONET/North (green).

a gap in 2004. Additional mid-eclipse times were published by Wood et al. (1993), Gürol \& Selan (1994), Wood \& Saffer (1999), Çakirli \& Devlen (1999), Kiss et al. (2000), Agerer \& Hübscher (2000, 2002, 2003), İbanoğlu et al. (2004), and Brát et al. (2008, 2009, 2011). Further timings were drawn from the Japanese VSNET archive (Kato et al. 2004) ${ }^{1}$, and unanalyzed light curves were obtained from the AAVSO archive (Henden 2010, priv. comm.) ${ }^{2}$. We determined mid-eclipse times for the AAVSO data, using the same fitting method as for the MONET data. The resulting new primary mid-eclipse times are included in Table 1.

The general picture that emerges from the entire body of eclipse times collected between 1984 and 2012 is that of a smooth long-term $\mathrm{O}-\mathrm{C}$ variation of complex shape (e.g. Kilkenny et al. 2003; İbanoǧlu et al. 2004; Lee et al. 2009). Our own observations demonstrate the absence of short-term $\mathrm{O}-\mathrm{C}$ variations with periods less than $1 \mathrm{yr}$ exceeding a couple of seconds. We have scrutinizingly surveyed the available data and found that a small number of published mid-eclipse times deviate significantly from the mean $\mathrm{O}-\mathrm{C}$ variation, suggesting that the errors were underestimated. Rather than including all data indiscriminately, we excluded these outliers from our analysis on the assumption that the absence of short-term $\mathrm{O}-\mathrm{C}$ variations as documented by the SAAO timings and our own data holds at all times. In general, we accepted mid-eclipse times if they have a quoted error not exceeding 0.0001 day. An adopted systematic error of $3.0 \mathrm{~s}$ was quadratically added to all eclipse times included in our data base. We restricted our analysis to primary mid-eclipse times, because the times for the secondary eclipses are significantly less well-determined and add little to the definition of the long-term $\mathrm{O}-\mathrm{C}$ variation. The mean phase of the secondary eclipses agrees with $\phi=0.500$ within a few seconds, similar to our finding for the sdB systems NSVS 14256825 and HS 0705+67 (Paper II).

Figure 1 shows the $\mathrm{O}-\mathrm{C}_{\text {lin,2 }}$ residuals of the data set adopted by us relative to the linear ephemeris used by Lee et al. (2009) in their Fig. 5 (top panel). We have included all 111 primary mideclipse times reported by the SAAO group, 20 from Lee et al. (2009), four from Wood et al. (1993) and Wood \& Saffer (1999), three from the OEJV (Brát et al. 2011), eight VSNET times,

\footnotetext{
1 http://www . kusastro. kyoto-u.ac.jp/vsnet/

2 http://www . aavso. org/ql
} 
Table 2. Parameters of the eclipsing binary HW Vir.

\begin{tabular}{lcc}
\hline \hline Parameter & Value \pm Error & Reference \\
\hline Orbital period $P_{\text {bin }}(\mathrm{s})$ & $10084.58 \pm 0.01$ & This work \\
Primary mass $M_{1}\left(M_{\odot}\right)$ & $0.485 \pm 0.013$ & Lee et al. (2009) \\
Secondary mass $M_{2}\left(M_{\odot}\right)$ & $0.142 \pm 0.004$ & Lee et al. (2009) \\
Secondary radius $R_{2}\left(R_{\odot}\right)$ & $0.175 \pm 0.026$ & Lee et al. (2009) \\
Separation $a_{\text {bin }}\left(R_{\odot}\right)$ & $0.860 \pm 0.010$ & Lee et al. (2009) \\
Inclination $i_{\text {bin }}\left({ }^{\circ}\right)$ & $80.9 \pm 0.1$ & Lee et al. (2009) \\
Eccentricity $e_{\text {bin }}$ & $<0.0003$ & This work \\
Distance $d(\mathrm{pc})$ & $181 \pm 20$ & Lee et al. (2009) \\
Visual magnitude $V(\mathrm{mag})$ & 10.9 & \\
\hline
\end{tabular}

three BAV times, and the new mid-eclipse times of Table 1. Our entire data base contains 176 primary mid-eclipse times. We have corrected all times to the Solar-system barycenter in the terrestrial system quoting them as $\mathrm{BJD}(\mathrm{TT})^{3}$.

In Table 2, we summarize the parameters of the binary HW Vir relevant to the present study. The masses and the distance are taken from Lee et al. (2009). The limit on the eccentricity was obtained from our new mid-eclipse times $T_{\text {ecl }}$ in Table 1, which limit the amplitude caused by apsidal motion to $\Delta T_{\text {ecl }} \simeq P_{\text {bin }} e / \pi<1.0 \mathrm{~s}$ for a period of the apsidal rotation $U \simeq P_{\text {bin }}\left(M_{2} / M_{1}\right)\left(a_{\text {bin }} / R_{2}\right)^{5} /\left(15 k_{2}\right) \simeq 43 \mathrm{~d}$, with $k_{2} \simeq 0.15$ the structure constant of the nearly fully convective secondary star (Feiden et al. 2011).

\section{The Lee et al. model}

In an influential paper, Lee et al. (2009) interpreted the mideclipse times of HW Vir available until 2008 (cycle number $E=76050$ ) by the LTT effect of two planets superposed on a quadratic variation of unspecified origin. Figure 1 shows their model curves for the quadratic variation (dashed curve) and their final model (solid curve). Their fit is adequate until 2008, but fails completely to reproduce our new data. The discrepancy has reached $250 \mathrm{~s}$ in 2012 and is largely due to the rapid fall-off of the quadratic term. The continuous period decrease as defined by the quadratic term does not exist.

The Lee et al. (2009) model faces the additional problem that the proposed 2-planet system is secularly unstable. This result is evident from the planetary parameters quoted by them, which imply crossing orbits with an apoapsis of the inner planet of $4.7 \mathrm{AU}$ and a periapsis of the outer planet of of 2.9 AU. We have numerically integrated the orbits with mercury6 (Chambers 1999, see below for more details) and find that a near encounter or a collision occurs within $2000 \mathrm{yr}$. Hence, the model of Lee et al. (2009) is untenable in the present form.

In the remainder of the paper, we show that all observations can be explained by the LTT effect, without taking recourse to an additional mechanism. A minor contribution by gravitational radiation is not excluded, but is below our detection limit.

\section{Search method}

We first scanned the two-companion parameter space for orbital periods below 60 years, using a generalized Lomb-Scargle periodogram supplemented by refined local searches. In a second step, we searched for improved solutions using a Levenberg-Marquardt (LM) minimization algorithm allowing us to detect the true local minima near the start parameters. Finally,

\footnotetext{
${ }^{3}$ http://astroutils.astronomy.ohio-state.edu/time/
}

we tested the secular stability of all possible solutions using the hybrid symplectic algorithm implemented in the mercury6 package (Chambers 1999).

Fitting Keplerian orbits to the set of mid-eclipse times involves a simultaneous fit for the ephemeris of the binary star (two parameters) and the orbits of the companions (five parameters each). The orbital inclination $i$ of a companion remains undetermined. For two or more companions, a direct search of the parameter space is computationally elaborate and time-consuming because of the large number of parameters involved. We adopted, therefore, the approach of Zechmeister \& Kürster (2009) and developed a variant of the generalized LombScargle (GLS) periodogram (Lomb 1976; Scargle 1982; Horne $\&$ Baliunas 1986). The mid-eclipse time of eclipse number $n$ including the LTT of planets $k=1 \ldots N_{\mathrm{p}}$ is expressed as

$T(n)=T_{0}+n P_{\text {bin }}+\sum_{k=1}^{N_{\mathrm{p}}} K_{k} \frac{1-e_{k}^{2}}{1+e_{k} \cos v_{k}(n)} \sin \left(v_{k}(n)+\omega_{k}\right)$,

where $e_{k}$ is the eccentricity of planet $k, K_{k}=a_{\mathrm{bin}, k} \sin i_{k} / c$ the amplitude of the LTT effect, with $a_{\text {bin, } k}$ the semi-major axis of the orbit of the center of gravity of the binary around the common center of mass and $i_{k}$ the inclination, $\omega_{k}$ is the argument of periastron measured from the ascending node in the plane of the sky, and $v_{k(n)}$ the true anomaly at time $t=T_{0}+n P_{\text {bin }}$ (Kopal 1959). Equation (2) can be transformed into a linear equation for the coefficients $A_{k}, B_{k}$, and $C$, using the eccentric anomaly $E_{k}(n)$ of planet $k$ at the time of eclipse $n$,

$T(n)=n P_{\text {bin }}+C+\sum_{k=1}^{N_{p}}\left(A_{k} \cos E_{k}(n)+B_{k} \sin E_{k}(n)\right)$

with

$A_{k}=K_{k} \sin \omega_{k}$

$B_{k}=K_{k} \sqrt{1-e_{k}^{2}} \cos \omega_{k}$

$C=T_{0}-\sum_{k=1}^{N_{\mathrm{p}}} K_{k} e_{k} \sin \omega_{k}$

The best-fit values of $T_{0}, P_{\text {bin }}, K_{k}$, and $\omega_{k}$ were derived by linear regression for given values of $e_{k}$, orbital period $P_{k}$ and time $t_{k}$ of periastron passage. Calculating a grid in these quantities reduces the number of free parameters for each grid point from $2+5 N_{\mathrm{p}}$ to $3 N_{\mathrm{p}}$.

In a second step, we searched for improved solutions near the best solution from GLS scan. We employed the LevenbergMarquardt (LM) minimization algorithm (Markwardt 2009), a non-linear least-squares fitting routine implemented in mpfit of IDL. We used the GLS-parameters as start values and generated 500 variations of them, using the diagonal elements of the mpfit-derived covariance matrix as uncertainties $\sigma$. The start parameters were varied randomly within $\pm 10 \sigma$ around the original start values, allowing us to detect the true local minimum near the start parameters.

Finally, we tested the secular stability of the solutions, using the hybrid symplectic integrator in mercury6 (Chambers 1999), which allows one to evolve planetary systems with high precision over long times very efficiently. We used constant time steps of $35 \mathrm{~d}$. Test runs demonstrated that this choice is adequate and smaller steps do not change the results. This provision is 


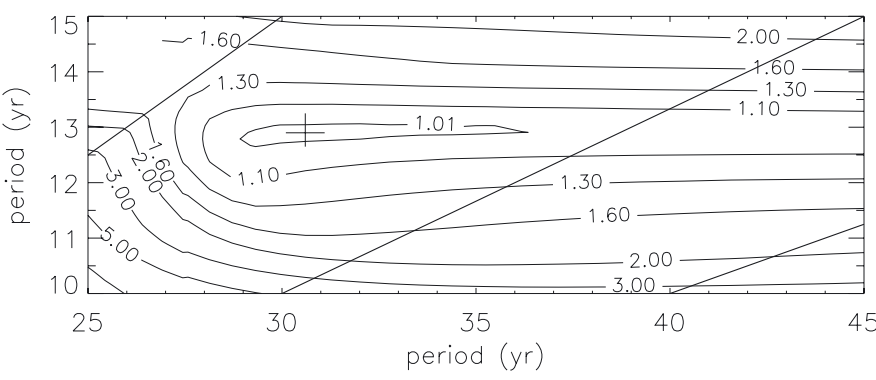

Fig. 2. Contour plot of the reduced $\chi^{2}$ normalized to unity for the best fit using a generalized Lomb-Scargle periodogram for a two-companion fit to the eclipse-time variations of HW Vir. The solid lines indicate 2:1, $3: 1$ and 4:1 resonant orbits, the cross the best fit. Solutions with an inner planet of about 12.7 year orbital period provide good fits for a wide range of orbital periods of the outer companion.

not adequate for the treatment of a close encounter, but such incidence should not occur in the successful models and if it does, the calculation is stopped and the model termed "unstable". The accuracy parameter in the code was set to $10^{-16}$, leading to a fractional change in the energy and momentum of the triple (binary and two companions) of typically $10^{-5}-10^{-4}$ and $10^{-9}-10^{-8}$, respectively. The quoted changes are valid for an integration time of $10^{7} \mathrm{yr}$ and the larger values obtain for longer periods $P_{4}$ and, hence, larger masses $M_{4}$. For simplicity, the central binary was treated as a single object with a mass equal to the sum of the component masses. This simplification is justified given the short binary orbital period of $2.8 \mathrm{~h}$. The gravitational field at the position of a distant companion can be represented as the sum of the constant field created by the combined mass of the binary components and a gravitational wave, emanating from the revolving binary with periods of $2.8 \mathrm{~h}$ for the fundamental and $1.4 \mathrm{~h}$ for the first harmonic. The relative strength of the wave field is $4 \times 10^{-8}$ and closely averages to zero over the 300 or 600 periods that occur in a time step of $35 \mathrm{~d}$. The retroaction of the companion tends to excite an eccentricity in the binary, but with a relative strength of $10^{-8}$ this effect is also entirely negligible. We used the masses of the binary components as given by Lee et al. (2009) and quoted in Table 2. The orbital evolution of the companions was followed until instability occurred, or at least for $10^{7} \mathrm{yr}$ and up to $10^{8} \mathrm{yr}$ for some models.

\section{A stable two-companion model for HW Vir}

The data shown in Fig. 1 suggest the presence of a period near 40000 cycles or $13 \mathrm{yr}$ superposed on a variation with a longer period. Using the GLS periodogram, we scanned the parameter space for two companions with orbital periods of $P_{3}=10-15$ yr and $P_{4} / P_{3} \geq 1.85$. Figure 2 shows a contour plot of the reduced $\chi^{2}$ normalized to unity for the best fit. For a better presentation, only the range $P_{4} \leq 45 \mathrm{yr}$ is displayed; no new features appear for larger $P_{4}$. A ridge of low $\chi^{2}$ confirms $P_{3} \simeq 12.5-12.9 \mathrm{yr}$ for all $P_{4} \gtrsim 28 \mathrm{yr}$, including the possible mean-motion resonances with $P_{4}: P_{3}=5: 2,3: 1,7: 2,4: 1$, and $5: 1$. None of the resonant solutions, however, is preferred over non-resonant ones. For the best fit near $P_{4}=30 \mathrm{yr}$, the inner object has a mass close to the boundary between planets and brown dwarfs, the outer one is a brown dwarf, with a mass increasing with orbital period up to the stellar mass limit for $P_{4} \simeq 70 \mathrm{yr}$.

With the LM optimization algorithm, we found improved two-companion solutions along the ridge of low $\chi^{2}$ in Fig. 2.
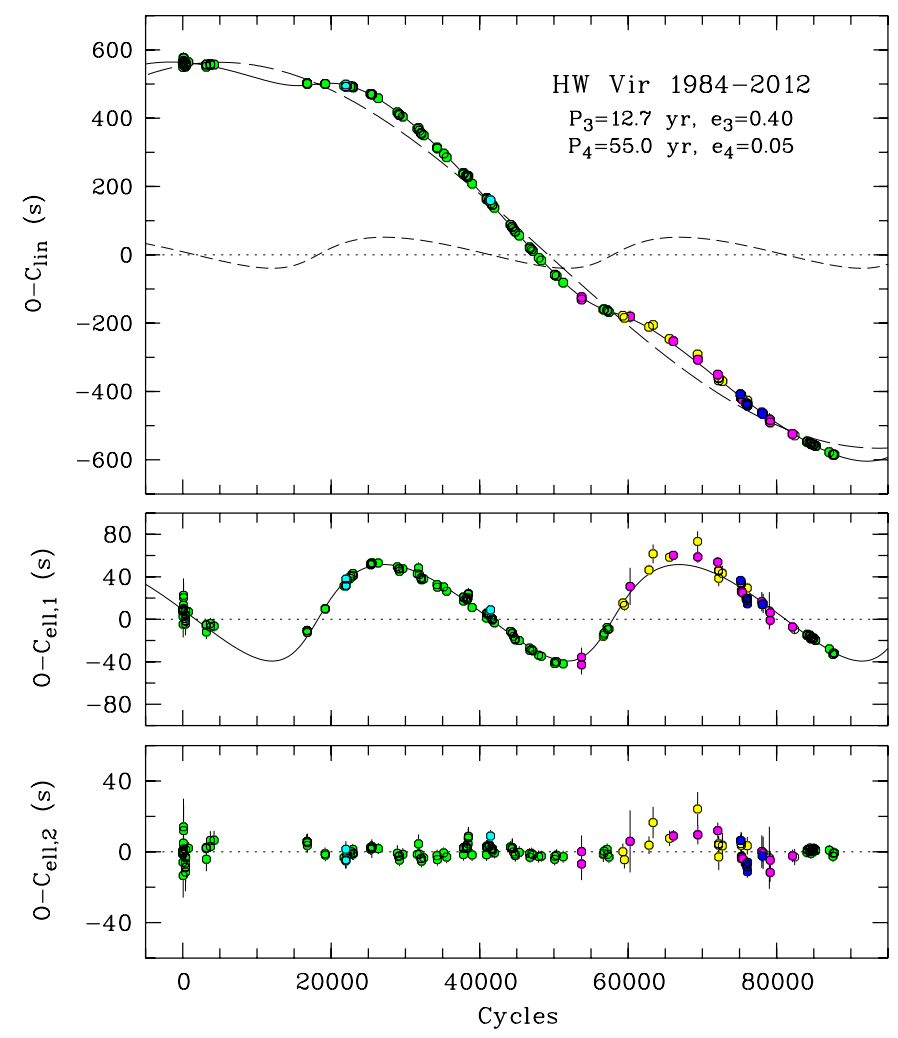

Fig. 3. Fit of two Keplerian orbits to the eclipse-time variations of HW Vir. Top: data of Fig. 1 relative to the linear ephemeris of Eq. (4). The curves denote the model LTT effect (solid) and the contributions by the outer companion (long dashes) and the inner planet (short dashes). Center: data with the contribution by the outer companion subtracted and model for the inner planet (solid curve). Bottom: residuals after the subtraction of the contributions by both companions.

Table 3. Parameters of the two-companion model of Fig. 3 for HW Vir.

\begin{tabular}{lcc}
\hline \hline Parameter & $\begin{array}{c}\text { Inner companion } \\
\# 3\end{array}$ & $\begin{array}{c}\text { Outer companion } \\
\# 4\end{array}$ \\
\hline Orbital period $P(\mathrm{yr})$ & $12.7 \pm 0.2$ & 55 (fixed) \\
Eccentricity $e$ & $0.40 \pm 0.10$ & $0.05:$ \\
Semi-major axis $a(\mathrm{AU})$ & $4.69 \pm 0.06$ & $12.8 \pm 0.2$ \\
Amplitude $K(\mathrm{~s})$ & $49 \pm 3$ & $563 \pm 200$ \\
Mass $M$ sin $i\left(M_{\text {Jup }}\right)$ & $14.3 \pm 1.0$ & $65 \pm 15$ \\
Argument of periastron $\omega\left(^{\circ}\right)$ & $-18 \pm 10$ & $0:$ \\
Periastron passage $(\mathrm{JD})$ & 2452401 & $2461677:$ \\
Periastron passage $(\mathrm{Cycle})$ & 57150 & $136619:$ \\
\hline
\end{tabular}

Notes. A colon indicates uncertain values.

Formally, the best fit is now obtained near a period ratio of $4: 1$ with periods $P_{3}=12.7 \mathrm{yr}$ and $P_{4}$ around $50 \mathrm{yr}$. The fit deteriorates for $P_{4}>70 \mathrm{yr}$. The eccentricity of the inner companion is rather well defined with $e_{3}=0.4 \pm 0.1$, that of the outer one is not, although small finite values fit better than $e_{4}=0$. Since the data cover only part of the orbital period of the outer companion, the deduced amplitude $K_{4}$ of the LTT effect depends on the adopted values of $P_{4}$ and $e_{4}$. Aided by the stability calculations described below, we chose of $P_{4}=55 \mathrm{yr}$ and $e_{4}=0.05$. Figure 3 (top panel) shows the best fit and Table 3 summarizes the fit results with our best estimates of the errors. The center panel repeats the data with the dominant contribution from the outer companion subtracted $\left(\mathrm{O}-\mathrm{C}_{\mathrm{ell}, 1}\right)$ and the bottom panel shows the residuals after subtraction of both 
K. Beuermann et al.: The quest for companions to post-common envelope binaries. III.
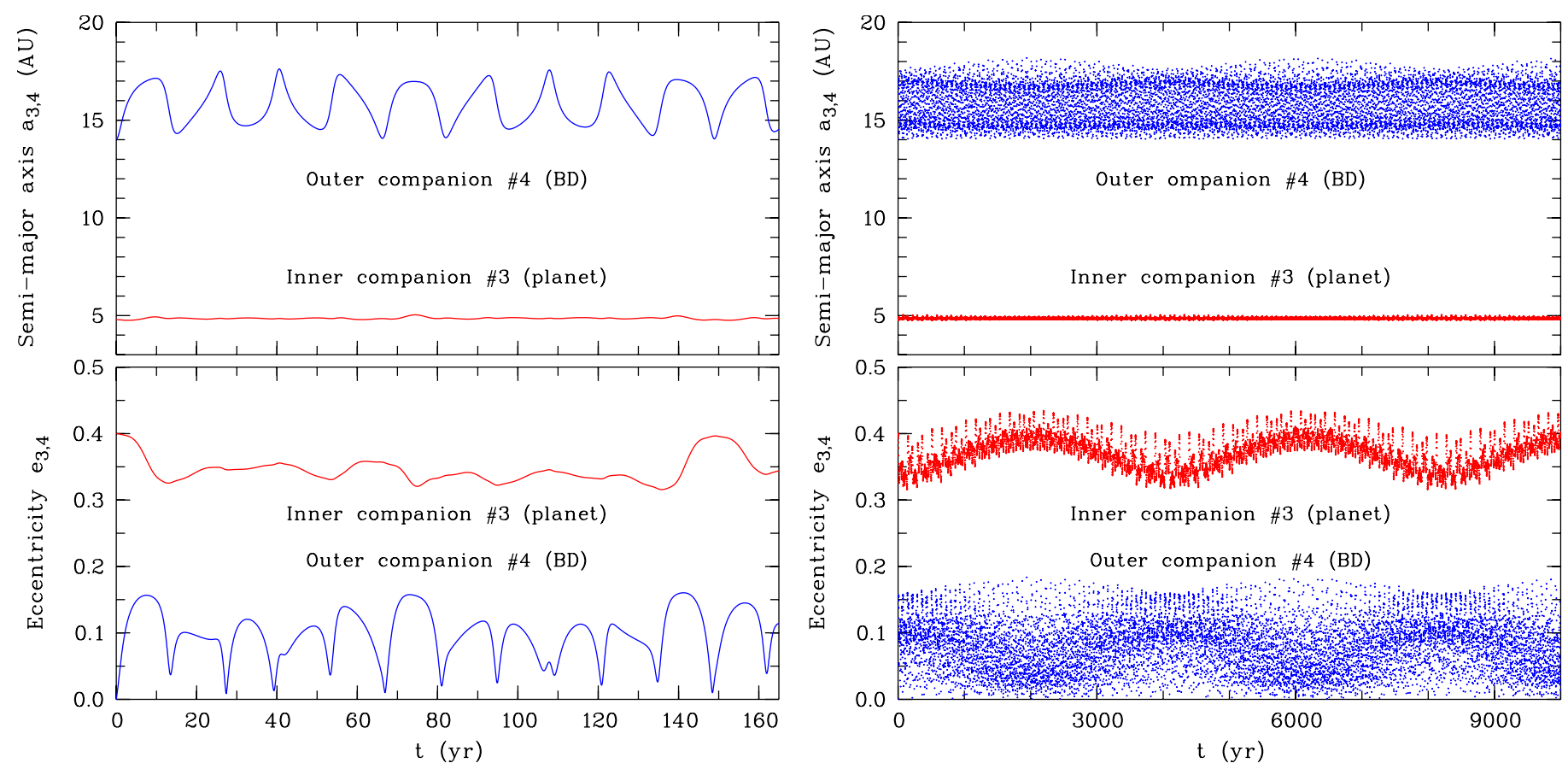

Fig. 4. Temporal variation of the semi-major axes and the eccentricities of the osculating orbits of the companions to HW Vir as calculated with the Mercury6 code (see text). The system is stable for more than $10^{8} \mathrm{yr}$ (see text). Color coding is the same for all panels.

contributions $\left(\mathrm{O}-\mathrm{C}_{\mathrm{ell}, 2}\right)$. Small systematic residuals exist, notably around cycle number 70000 , where the data quality is not the best, however. The formal best fit leads to a symmetric configuration with $\Delta \omega=\omega_{4}-\omega_{3}$ near zero, but with a large uncertainty in $\omega_{4}$, because of the limited data coverage. The general decrease of $\mathrm{O}-\mathrm{C}_{\text {lin }}$ over the 1984-2012 time interval reflects the fact that the observations cover only that part of the orbit, when the outer companion is receding from the observer and the binary is approaching. The corresponding LTT amplitude suggests that the outer companion is probably a brown dwarf with a mass of roughly $65 M_{\text {Jup }}$ or a star of very low mass. For periods between 40 and $70 \mathrm{yr}$, its mass would be in the range of 30 to $120 M_{\text {Jup }}$. The fit yields an underlying linear ephemeris of the binary

$$
T_{\mathrm{ecl}}=\mathrm{BJD}(\mathrm{TT}) 2445730.5497(72)+0.11671969(15) E,
$$

where the quoted correlated uncertainties of the epoch and the binary period $P_{\text {bin }}$ refer to the quoted range of $P_{4}$.

Using the mercury 6 code, we followed the orbital evolution of solutions along the low- $\chi^{2}$ ridge in Fig. 2 numerically, using combinations of periods $P_{3}$ and $P_{4}$ and masses $M_{3}$ and $M_{4}$ that match the observations. We note that the actual positions of the two companions in space, their orbital velocities, and true anomalies at a given time can not be obtained with sufficient accuracy from the observations. We employed, therefore, standardized start parameters and searched for stable models that reproduce the observations for a substantial percentage of the time. The calculations were started in either the symmetric or the antisymmetric configuration with both companions at periastron, having Keplerian velocities. The orbits were taken to be coplanar with the binary orbit $\left(i=80.9^{\circ}\right.$, Table 2$)$, with the start eccentricities as free parameters. Orbital evolution changes the elements quickly and periodically varying eccentricities develop also for models that start with circular orbits.

We dubbed a model "stable" if it persisted without close encounter for more that $10^{7} \mathrm{yr}$. Such models are found for periods $P_{4}$ as short as $35 \mathrm{yr}$, but for most start configurations at this period instability incurred, sometimes only after $10^{6} \mathrm{yr}$. The fraction of stable models that mimic the observations becomes substantial only near $45 \mathrm{yr}$ and at $55 \mathrm{yr}$ or longer. A low fraction of stable models near $37 \mathrm{yr}$ and, less so, near $50 \mathrm{yr}$ may imply a higher probability of instability near the $3: 1$ and 4:1 mean-motion resonances. The general preference for longer periods $P_{4}$ is expected, because the observations fix $P_{3}$ between 12.5 and $12.9 \mathrm{yr}$ and a more distant outer companion increases the phase space for solutions with larger eccentricities. At $P_{4} \geq 55 \mathrm{yr}$, stable models were found for eccentricities $e_{3}$ as large as 0.45 . The properties of stable models between $P_{4}=45 \mathrm{yr}$ and $55 \mathrm{yr}$ differ in that the former prefer antisymmetric configurations, whereas the latter all spend a substantial fraction of the time near a symmetric configuration. Taken at face value, our observational best fit of $\Delta \omega$ near zero favors a model with $P_{4}>50 \mathrm{yr}$. Our preferred model has $P_{4}=55 \mathrm{yr}$, values of $P_{3}, e_{3}, M_{3}$, and $M_{4}$ as obtained from the fit to the data (Fig. 3, Table 3), and orbits coplanar with the binary.

Figure 4 shows the evolution of the semi-major axes and eccentricities for the preferred model. The left panels illustrate the behavior for the first $165 \mathrm{yr}$ (three orbital periods of the outer companion), the right panels show the evolution over $10000 \mathrm{yr}$. The system is stable for $10^{8} \mathrm{yr}$. The elements of the osculating orbits vary periodically on time scales up to $4000 \mathrm{yr}$ (right panels). The eccentricities $e_{3}$ and $e_{4}$ range from 0.32 to 0.43 and from zero to 0.18 , respectively. Over short time intervals, the modulation at the synodic period of the inner planet $P_{\text {syn }}=$ $\left(P_{3}^{-1}-P_{4}^{-1}\right)^{-1} \simeq 16 \mathrm{yr}$ is dominant (left panels). The system spends a substantial fraction of the time near the observed eccentricities, $e_{3} \simeq 0.4$ and $e_{4} \simeq 0.05$, and assumes a symmetric configuration most frequently near maximum $e_{3}$ in the $4000 \mathrm{yr}$ cycle (lower right panel), suggesting that the observational fit of Fig. 3 and Table 3 represents a snapshot of the variable constitution of the HW Vir system. While the agreement seems satisfactory, we add the caveat that our simulations have so far only provided a first glimpse at the stability landscape, which remains to be explored in more detail. 
The perturbation of the orbits by the mutual interaction of the two companions leads to non-Keplerian shifts of the observed eclipse times, which are not included in the fit shown in Fig. 3. Our simulations show that such shifts are not negligible and affect the derived values of the LTT amplitude, the period, the eccentricity, and the argument of periastron of both companions. In particular, the LTT amplitude of the inner companion can vary by more than $10 \mathrm{~s}$ between subsequent orbits. Such orbit-to-orbit variations are not correctly interpreted by our present fitting routines. The observed residuals $\mathrm{O}-\mathrm{C}_{\text {ell, } 2}$ after subtraction of the contributions from the two Keplerian contributions may show a non-random structure, possibly representing the signature of the perturbed orbits (Fig. 3, bottom panel), but the quality of the data is lowest between cycle numbers 60000 and 80000 , where the excursion appears largest. Clearly, a unique identification of non-Keplerian eclipse-time variations in PCEB would provide a strong support for the planetary model.

We have alternatively considered that the residuals in Fig. 3 might indicate the presence of a third inner planet. While the added free parameters improve the fit, this possibility can be excluded, because such object is quickly expelled.

\section{Conclusion}

We have investigated the plausibility of the planetary model for HW Vir, using additional data and an exhaustive search for and dynamical tests of two-companion solutions. The original model by Lee et al. (2009) is clearly ruled out, both from the current direction of the observed $\mathrm{O}-\mathrm{C}$ variations as well as the fact that their solution is dynamically unstable. We have presented a qualitatively different solution that involves two companions in secularly stable orbits about the binary HW Vir. The inner companion has an orbital period $P_{3} \simeq 12.7 \pm 0.2 \mathrm{yr}$ and a mass $M_{3} \simeq 14.3 \pm 1.0 M_{\text {Jup }}$ for an inclination $i_{3}$ identical to that of the binary ( $i=80.9^{\circ}$, see Table 2$)$. For other inclinations, the mass varies as $1 / \sin i_{3}$. The outer more massive companion is a brown dwarf or a low-mass star with a mass around $M_{4} \sin i_{4} \simeq 30$ to $120 M_{\text {Jup }}$, where $i_{4}$ is the unknown inclination of its orbit. Its parameters are still uncertain, because the data cover only about half an orbital period. While the suggested pair of companions in coplanar orbits is secularly stable, we caution that the stability landscape has not been thoroughly explored and its systematic structure is still eluding us. The system is strongly interacting with evidence for non-Keplerian orbits, whose signature may even be visible in the data.

Our model of HW Vir provides an attractive possibility of explaining the entire observed orbital-period variations of HW Vir by the LTT effect, without taking recourse to any additional unexplained process. It provides positive evidence of the planetary model for HW Vir and strengthens the case for close binaries in general. It also calls for a re-examination of other systems in the light of our result. However, the case of HW Vir also shows how difficult it may be to find such a solution, given both the very long time-series required to detect clearly periodic planetary signals, the requirement of fitting all residuals without invoking other arbitrary mechanisms, and the limits of fitting individual static Keplerian orbits to what must obviously be very dynamic systems.
Acknowledgements. We thank the anonymous referee for helpful comments that improved the presentation. This work is based in part on data obtained with the MOnitoring NEtwork of Telescopes (MONET), funded by the Alfried Krupp von Bohlen und Halbach Foundation, Essen, and operated by the Georg-AugustUniversität Göttingen, the McDonald Observatory of the University of Texas at Austin, and the South African Astronomical Observatory. We acknowledge with thanks the variable star observations from the AAVSO International Database contributed by observers worldwide and used in this research. The work also benefitted from the use of data made available by the Variable Star Network, World Center for Transient Object Astronomy and Variable Stars. Finally, we thank Tim-Oliver Husser for measuring the eclipse on 21 January 2011.

\section{References}

Agerer, F., \& Hübscher, J. 2000, IBVS, 4912, 1

Agerer, F., \& Hubscher, J. 2002, IBVS, 5296, 1

Agerer, F., \& Hübscher, J. 2003, IBVS, 5484, 1

Applegate, J. H. 1992, ApJ, 385, 621

Beuermann, K., Hessman, F. V., Dreizler, S., et al. 2010, A\&A, 521, L60

Beuermann, K., Buhlmann, J., Diese, J., et al. 2011, A\&A, 526, A53

Beuermann, K., Breitenstein, P., Dẹbski, B. D., et al. 2012, A\&A, 540, A8 (Paper II)

Brát, L., Šmelcer, L., Kuèáková, H., et al. 2008, OEJVS, 94, 1

Brát, L., Trnka, J., Lehky, M., et al. 2009, OEJVS, 107, 1

Brát, L., Trnka, J., Šmelcer, L., et al. 2011, OEJVS, 137, 1

Brinkworth, C. S., Marsh, T. R., Dhillon, V. S., \& Knigge, C. 2006, MNRAS, 365,287

Çakirli, Ö., \& Devlen, A. 1999, A\&AS, 136, 27

Chambers, J. E. 1999, MNRAS, 304, 793

Doyle, L. R., Carter, J. A., Fabrycky, D. C., et al. 2011, Science, 333, 1602

Feiden, G. A., Chaboyer, B., \& Dotter, A. 2011, ApJ, 740, L25

Gürol, B., \& Selan, S. 1994, IBVS, 4109, 1

Hinse, T. C., Lee, J. W., Goździewski, K., et al. 2012, MNRAS, 420, 3609

Horne, J. H., \& Baliunas, S. L. 1986, ApJ, 302, 757

Horner, J., Marshall, J. P., Wittenmyer, R. A., \& Tinney, C. G. 2011, MNRAS, 416, L11

İbanoğlu, C., Çakırlı, Ö., Taş, G., \& Evren, S. 2004, A\&A, 414, 1043

Kato, T., Uemura, M., Ishioka, R., et al. 2004, PASJ, 56, 1

Kilkenny, D., Harrop-Allin, M., \& Marang, F. 1991, IBVS, 3569, 1

Kilkenny, D., Marang, F., \& Menzies, J. W. 1994, MNRAS, 267, 535

Kilkenny, D., Keuris, S., Marang, F., et al. 2000, The Observatory, 120, 48

Kilkenny, D., van Wyk, F., \& Marang, F. 2003, The Observatory, 123, 31

Kiss, L. L., Csák, B., Szatmáry, K., Furész, G., \& Sziládi, K. 2000, A\&A, 364, 199

Kopal, Z. 1959, Close Binary Systems (Chapman \& Hall), 109

Lee, J. W., Kim, S.-L., Kim, C.-H., et al. 2009, AJ, 137, 3181

Lomb, N. R. 1976, Ap\&SS, 39, 447

Lovis, C., Ségransan, D., Mayor, M., et al. 2011, A\&A, 528, A112

Marang, F., \& Kilkenny, D. 1989, IBVS, 3390, 1

Markwardt, C. B. 2009, ASP Conf. Ser., 411, 251

Nather, R. E., \& Robinson, E. L. 1974, ApJ, 190, 637

Parsons, S. G., Marsh, T. R., Copperwheat, C. M., et al. 2010b, MNRAS, 407, 2362

Potter, S. B., Romero-Colmenero, E., Ramsay, G., et al. 2011, MNRAS, 416, 2202

Qian, S.-B., Zhu, L. Y., Zola, S., et al. 2009, ApJ, 695, L163

Qian, S.-B., Zhu, L.-Y., Liu, L., et al. 2010a, Ap\&SS, 329, 113

Qian, S.-B., Dai, Z.-B., Liao, W.-P., et al. 2010b, ApJ, 708, L66

Qian, S.-B., Liu, L., Liao, W.-P., et al. 2011, MNRAS, 414, L16

Qian, S.-B., Liu, L., Zhu, L.-Y., et al. 2012a, MNRAS, 422, L24

Qian, S.-B., Zhu, L.-Y., Dai, Z.-B., et al. 2012b, ApJ, 745, L23

Scargle, J. D. 1982, ApJ, 263, 835

Todoran, I. 1972, Ap\&SS, 15, 229

Watson, C. A., \& Marsh, T. R. 2010, MNRAS, 405, 2037

Welsh, W. F., Orosz, J. A., Carter, J. A., et al. 2012, Nature, 481, 475

Wittenmyer, R. A., Horner, J. A., Marshall, J. P., Butters, O. W., \& Tinney, C. G. 2012, MNRAS, 419, 3258

Wood, J. H., \& Saffer, R. 1999, MNRAS, 305, 820

Wood, J. H., Zhang, E.-H., \& Robinson, E. L. 1993, MNRAS, 261, 103

Zechmeister, M., \& Kürster, M. 2009, A\&A, 496, 577 